\title{
Tocilizumab for COVID-19: A systematic review and meta-analysis of randomized controlled trials
}

\author{
Tarun Krishna Boppana, Saurabh Mittal, Karan Madan, Anant Mohan, Vijay Hadda, Randeep Guleria \\ Department of Pulmonary, Critical Care and Sleep Medicine, All India Institute of Medical Sciences (AIIMS), New Delhi, \\ India
}

\begin{abstract}
The effective treatment modalities for severe coronavirus disease 2019 (COVID-19) are needed. As the primary cause of mortality is a hyperinflammatory state, the interleukin-6 antagonist tocilizumab has been used in multiple clinical studies. We conducted this systematic review and meta-analysis to estimate the effectiveness of tocilizumab in reduction of mortality due to COVID-
\end{abstract}

Correspondence: Dr. Saurabh Mittal, Department of Pulmonary, Critical Care and Sleep Medicine, All India Institute of Medical Sciences (AIIMS), Ansari Nagar, New Delhi 110029, India. Tel. +91.11.26546357.

E-mail: saurabh_kgmu@yahoo.co.in - saurabh.aiims@aiims.edu

Key words: tocilizumab; IL-6 inhibitor; interleukin 6; COVID-19; SARS-CoV-2; meta-analysis.

Contributions: TKB, literature search, manuscript preparation; SM, conception, literature search, manuscript revision; KM, VH, AM, RG, manuscript revision. All the authors have read and approved the final version of the manuscript and agreed to be accountable for all aspects of the work.

Conflict of interest: The authors declare that they have no competing interests, and all authors confirm accuracy.

Ethics approval: Not applicable.

Availability of data and material: All data are available from the corresponding author on reasonable request.

PROSPERO registration number: CRD42021258091

Received for publication: 24 October 2021.

Accepted for publication: 17 January 2022.

Publisher's note: All claims expressed in this article are solely those of the authors and do not necessarily represent those of their affiliated organizations, or those of the publisher, the editors and the reviewers. Any product that may be evaluated in this article or claim that may be made by its manufacturer is not guaranteed or endorsed by the publisher.

${ }^{\circ}$ Copyright: the Author(s), 2022

Licensee PAGEPress, Italy

Monaldi Archives for Chest Disease 2022; $92: 2136$

doi: 10.4081/monaldi.2022.2136

This article is distributed under the terms of the Creative Commons Attribution-NonCommercial International License (CC BY-NC 4.0) which permits any noncommercial use, distribution, and reproduction in any medium, provided the original author(s) and source are credited.
19. A systematic search of the PubMed and Embase databases was performed to extract randomized controlled trials (RCTs) regarding the use of tocilizumab therapy for COVID-19. An overall pooled mortality analysis was performed, and odds ratios were reported. Cochrane risk of bias assessment tool was used to assess the risk of bias. Heterogeneity was assessed using the $\mathrm{I}^{2}$ statistic. Nine RCTs, including 6489 patients, were selected for meta-analysis. Seven trials reported 28-day mortality, and one trial each reported 21-day and 30-day mortality. There were 846 deaths among 3358 participants in the tocilizumab group while 943 deaths among 3131 patients randomized to the control group (random-effects odds ratio $0.87,95 \%$ confidence interval $0.73-1.03, \mathrm{p}=0.11$ ). There was some heterogeneity among the trials as the $\mathrm{I}^{2}$ value was $15 \%$, with a $\mathrm{p}$ value of 0.31 . There was a reduction in the need for ICU admission in the tocilizumab group. A higher risk of secondary infections was noted in the tocilizumab group (fixed-effects odds ratio $0.72,95 \%$ confidence interval $0.55-0.95, \mathrm{p}=0.02$ ). This meta-analysis of RCTs demonstrated that the use of tocilizumab was not associated with a reduction in all-cause mortality in patients with COVID-19 and had higher odds of secondary infections.

\section{Introduction}

Coronavirus disease 2019 (COVID-19) has affected the world over the last year and has led to a significant burden on healthcare systems globally [1]. The disease varies in severity from mild infection to critical illness and is associated with high mortality in patients requiring hospitalization and intensive care unit (ICU) admission. Since the beginning of the pandemic, clinicians and scientists have been looking for treatment options for the illness. Many classes of drugs are being repurposed to treat COVID-19 [2,3]. To date, the most beneficial agent for preventing mortality from the disease has been steroids. Awake proning has also been shown to be a promising modality $[4,5]$. It has now been well understood that virus-induced inflammation is the key driver of lung injury and probably contributes significantly to disease-related mortality. Due to this, the drugs which inhibit the inflammatory pathways are being tried for use in severe COVID-19. Interleukin-6 (IL-6) levels have been seen consistently elevated in COVID-19; however, the levels are much lower than the levels seen in many other inflammatory and infectious diseases. The IL-6 inhibitor tocilizumab has been in clinical use in immunological disorders for many years. It is one of the several agents being tried for COVID-19. Initially, multiple case reports and case series had shown the benefits of tocilizumab therapy for COVID-19 [6]. However, since then, many randomized clinical trials have been published, and the majority have not shown any significant mortality benefit with the use of tocilizumab. 
Other anti-IL-6 therapies, sarilumab and situximab, have also been used for COVID-19 and have shown similar results [7,8]. One primary concern with the use of these immunosuppressive agents has been the risk of secondary infections. The largest trial available regarding the use of tocilizumab in COVID-19 is the RECOVERY (Randomized Evaluation of COVID-19 Therapy) trial which demonstrated a mortality benefit using tocilizumab therapy [9]. However, results from other trials have been variable [10-12]. Multiple meta-analyses regarding tocilizumab use in COVID-19, including retrospective studies, have been done $[13,14]$. However, due to continuously emerging evidence, the actual benefit of this agent in reducing mortality or any other outcomes remains unclear. Hence, we conducted this systematic review and meta-analysis to analyze the current evidence for the efficacy of tocilizumab therapy in reducing mortality in patients with COVID-19.

\section{Methods}

This systematic review was reported according to the Preferred Reporting Items for Systematic Reviews and Meta-Analyses (PRISMA) statement [15].

\section{Eligibility criteria}

We included randomized controlled trials involving adults with COVID-19 wherein tocilizumab was compared with placebo or standard of care. All single-arm studies, non-randomized prospective and retrospective studies were excluded. The trials reporting mortality at day 28-30 were included in the meta-analysis.

\section{Search strategy and initial review}

Two investigators (SM and TKB) performed a systematic search of the two databases PubMed and EMBASE, to identify the original, peer-reviewed, full-length, human subject articles describing the use of tocilizumab to treat COVID-19. The following database-specific Boolean search strategy was used. Free text search terms were: (COVID OR coronavirus disease) AND ("tocilizumab" OR "IL-6 antagonist” OR “interleukin-6 antagonist”).

The study protocol was registered and made available on the PROSPERO database (CRD42021258091) on June 1st, 2021. All retrieved articles were imported into reference management software. Following the removal of duplicate citations, the initial screening of the studies was performed by title and abstract. For all relevant articles, full texts were downloaded for review. The reference lists of the extracted articles were also reviewed to look for potential studies. The finally selected studies were independently screened by two authors (SM and TKB). We included only original articles describing the results of a randomized controlled trial regarding tocilizumab in COVID-19 and reporting mortality outcomes.

\section{Data abstraction}

Following careful review of the selected articles, the data were extracted on the data extraction form. The following information was retrieved after a thorough review of the full text: i) author, ii) year, iii) number of patients in drug and control group, iv) study country, v) inclusion criteria, vi) dose of tocilizumab used, vii) oxygenation status at randomization, viii) proportion of patients on mechanical ventilation, ix) C-reactive protein levels, x) IL-6 levels, xi) mortality in each group xii) primary outcome of the trial, xiii) mortality in ICU patients, xiv) need for ICU admission and $\mathrm{xv}$ ) occurrence of secondary infections in each group. The systematic review methodology is shown in Figure 1.

The primary outcome analyzed was the day 28-30 mortality between the two groups. We also performed a subgroup analysis to assess the mortality difference between patients admitted to ICU at baseline. The other outcome assessed during the review was the need for ICU admission among patients not admitted to ICU at enrollment. Safety outcomes included the incidence of secondary infections in each group.

\section{Risk of bias assessment}

We used the Cochrane Risk of Bias Assessment tool to assess the risk of bias in each trial (reported as low risk, high risk or unclear risk). The following criteria were used to assess the risk of bias: The generation of randomization sequence and allocation concealment, blinding, and completeness of the data and reporting of outcomes. Two authors (SM and TKB) completed these independently, and disagreements were resolved by mutual discussion.

\section{Statistical analysis}

Statistical analyses were performed using the STATA statistical analysis software (StataCorp. 2017, Release 15. StataCorp LLC., College Station, TX, USA), and forest plots were generated using Revman 5.0. The primary analysis was done by the inverse-variance method with a random-effects model reporting the odds ratio for overall mortality. For trials reporting mortality in patients admitted to ICU, a similar analysis to assess mortality was performed. Fixed-effects model analysis was also done.

The impact of heterogeneity on the pooled estimates of the outcome was assessed using the $\mathrm{I}^{2}$ statistic, and $\mathrm{p}$ values were generated using the Cochran Q statistic. Publication bias was assessed by funnel plot and Egger's test (statistically significant publication bias if $\mathrm{p}<0.1$ ). The meta-regression was done according to baseline CRP levels.

\section{Results}

The initial literature search yielded 1543 articles, and 1073 results were obtained after duplicate removal, from which 11 published randomized controlled trials were selected. One article found was a follow-up data of the included RCT, and one article by Zhao et al. did not report mortality outcome [16,17]. So, finally, nine articles were included for detailed review and meta-analysis. One article reported day 21 mortality rather than day 28-30, and it was used for meta-analysis [18]. The flow diagram depicting the identification of eligible trials for the meta-analysis is shown in Figure 1. The included studies were conducted in the United States of America, the United Kingdom, Brazil, India and multiple 
European countries, including France and Italy [9-12,18-22]. The basic details of the included trials are summarized in Table 1.

The Randomized, Embedded, Multifactorial Adaptive Platform Trial for Community-Acquired Pneumonia (REMAPCAP) trial randomized patients to tocilizumab and Sarilumab; however, we included data only from patients who received tocilizumab [18]. The total number of patients randomized in the nine trials was 6489 (3358 to tocilizumab group and 3131 to placebo or standard of care). About 2253 among cases, while 2140 among controls were males. All trials included patients on some respiratory support. Among cases, 499 were on IMV, while among controls, 483 were receiving invasive mechanical ventilation at enrollment. The risk of bias was low in four trials, while five trials had some concerns (Figure 2).

\section{Study outcomes}

The outcome measures from individual trials are summarized in Table 2. There were 846 deaths among 3358 participants randomized to tocilizumab therapy $(25.19 \%)$, while in the control group, 943 participants died among 3131 randomized $(30.11 \%)$. Based on a random-effect meta-analysis, the summary odds ratio was $0.87(95 \%$ confidence interval $0.73-1.03, \mathrm{p}=0.11)$ for all-cause mortality comparing tocilizumab with placebo or standard of care (Figure 3). The summary OR using a fixed-effect model for metaanalysis was $0.84(95 \% \mathrm{CI}, 0.75-0.94)$. The overall inverse variance-weighted fixed-effect risk ratio was 0.88 (95\% CI, 0.82-0.96, $\mathrm{p}=0.002$ ) for all-cause mortality among all randomized patients.

\section{Databases searched: Pubmed and Embase
Search terms: (COVID OR coronavirus disease) AND ("tocilizumab" OR "IL-6 antagonist"
OR "interleukin-6 antagonist")}
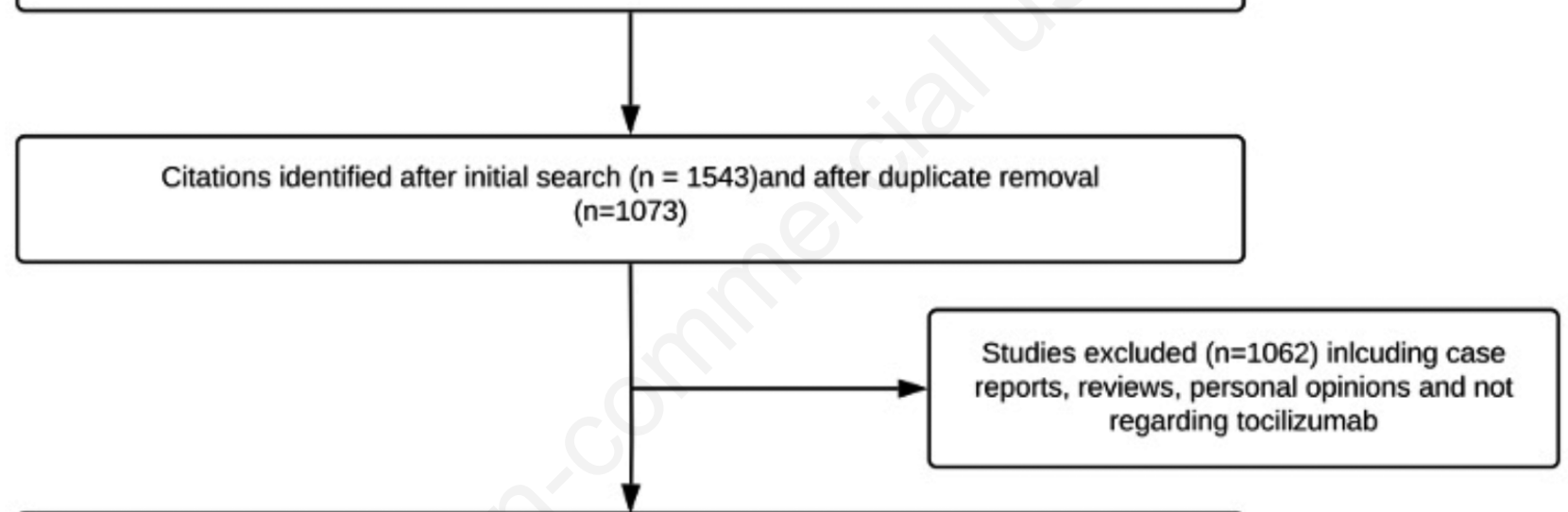

Randomized controlled trials related to use of tocilizumab in COVID-19 $(n=11)$

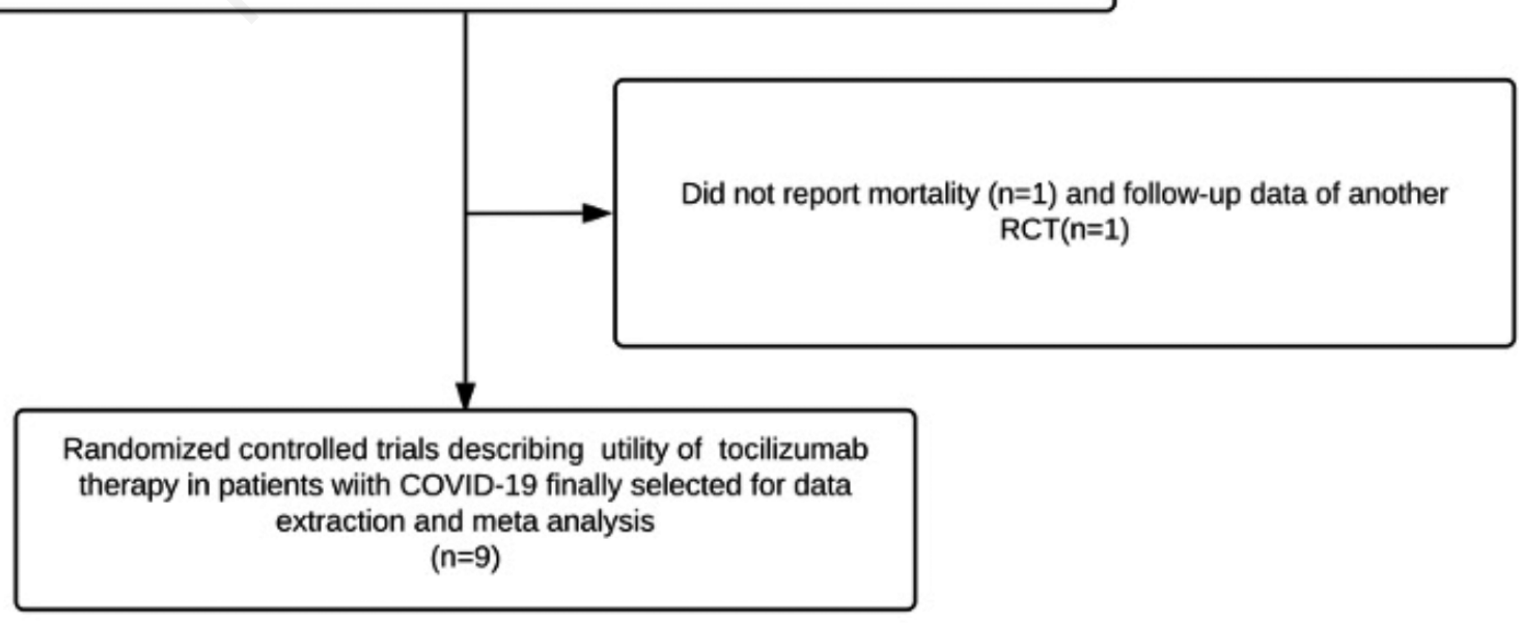

Figure 1. Flow diagram describing the process of systematic review and selection of relevant studies for meta-analysis. 


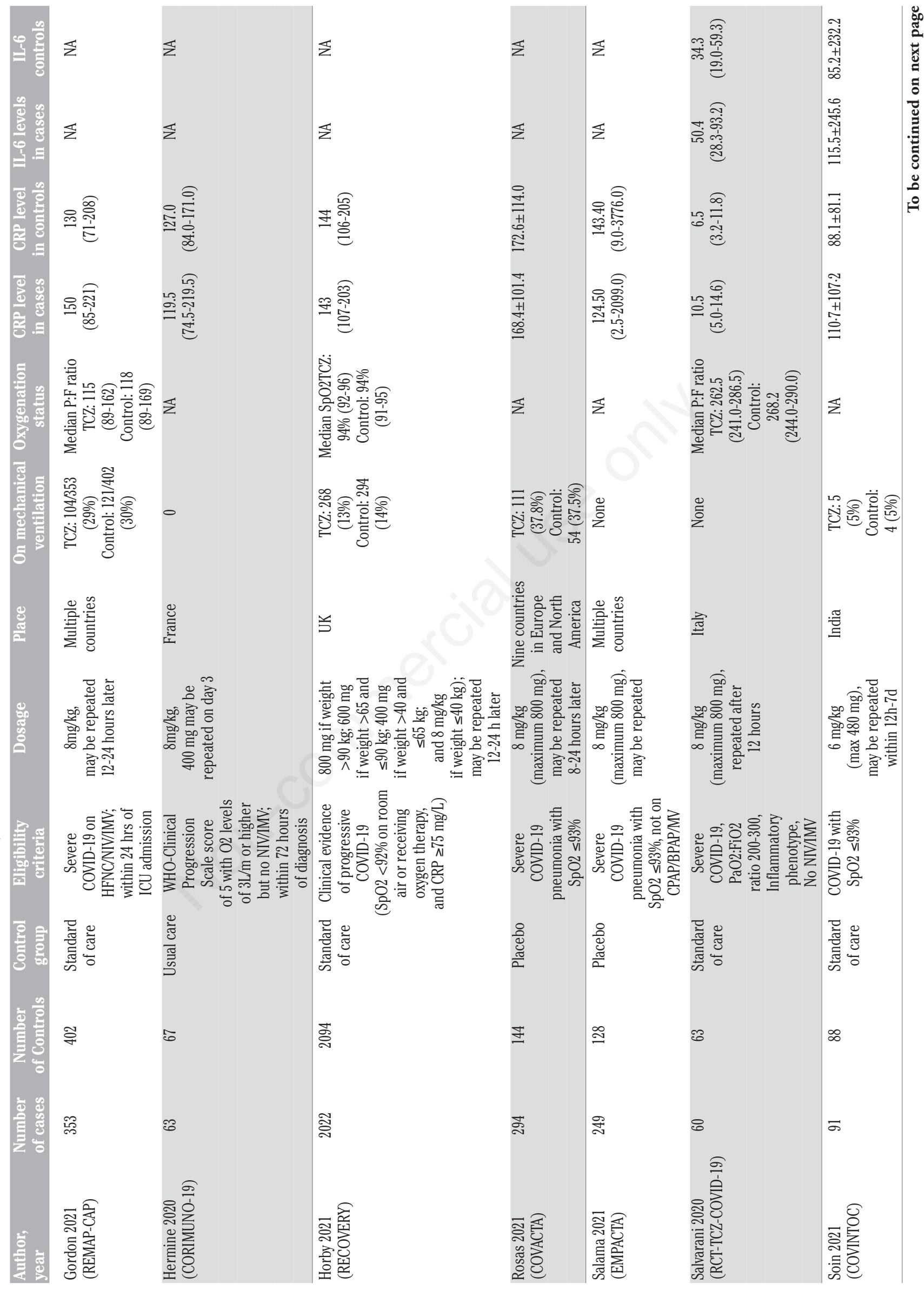




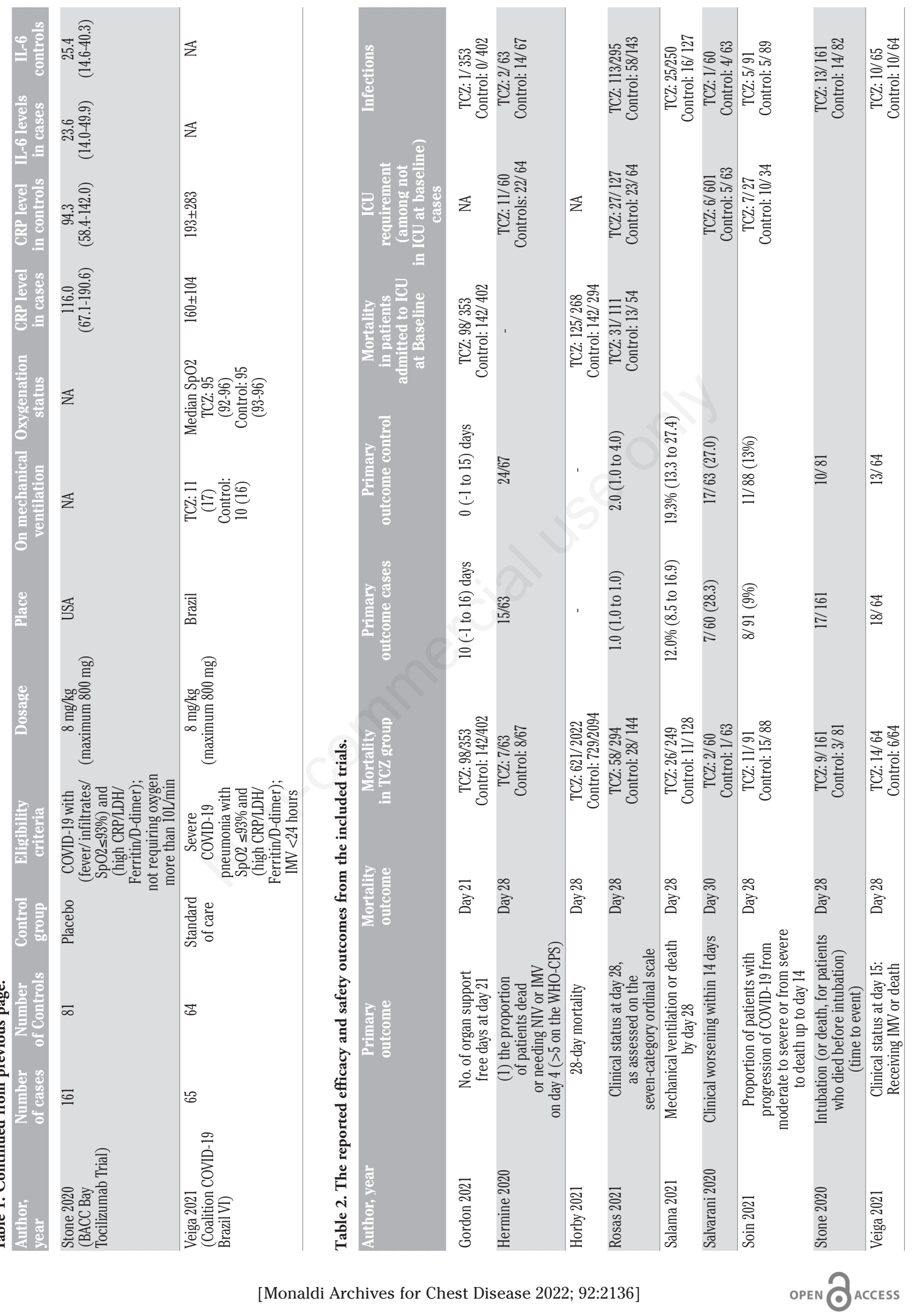


Three trials reported the mortality separately for patients who were admitted to ICU at enrollment $[9,18,20]$. The inverse variance-weighted random-effect odds ratio for mortality in this subgroup was $0.84(95 \% \mathrm{CI}, 0.65-1.10, \mathrm{p}<0.20)$ (Figure 4). Among patients who were not admitted to ICU at baseline, we assessed the need for ICU admission among these. Four trials reported this outcome, and 51 out of 274 in the tocilizumab group and 60 out of 225 in the control group required ICU admission $[10,12,19,20]$. The summary OR for this outcome was 0.57 (95\% CI, 0.37-0.89), suggesting a significant reduction in the need for ICU admission in the tocilizumab group (Figure 5). On meta-regression, there was no evidence of a relationship with baseline CRP (logOR versus baseline CRP; $p=0.41$ ) (Supplementary Figure 1). The subgroup analysis bases upon hyperinflammatory phenotype at admission was

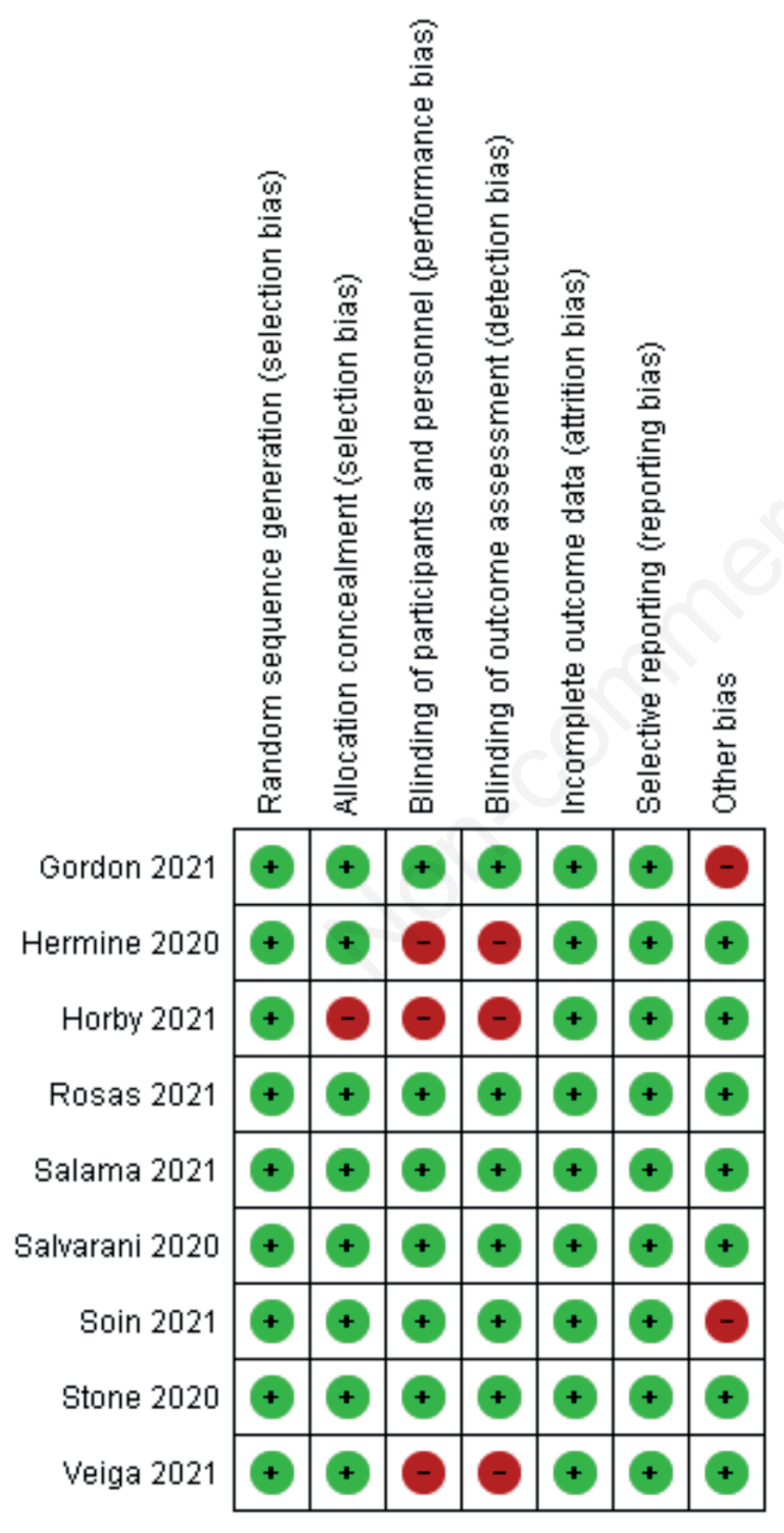

Figure 2. The risk of bias assessment summary using Cochrane Risk of Bias Assessment tool. +, low risk of bias; -, high risk of bias. also performed but did not reveal any significant difference between the groups (Supplementary Figure 2). There was some degree of heterogeneity among the trials as the $\mathrm{I}^{2}$ value was $15 \%$, with a $p$-value of 0.31 . There was no evidence of publication bias on the visual inspection of the funnel plot as well as by Egger's test $(\mathrm{p}=0.20)($ Supplementary Figure 3$)$.

\section{Safety assessment}

Among the nine included studies, one did not report the absolute number of secondary infections, and it was reported as not significantly different between the two groups [9]. In the other eight RCTs, 170 events occurred among 1338 patients randomized to tocilizumab (12.7\%), and 121 events occurred among 1037 patients randomized to standard of care or placebo (11.6\%). The fixed-effect summary OR was $0.72(95 \% \mathrm{CI}, 0.55-0.95, \mathrm{p}=0.02)$, and there was some inconsistency between the trial results (I2= $33 \%, p=0.17$ for heterogeneity; Figure 6).

\section{Discussion}

In this systematic review and meta-analysis of nine randomized controlled trials, including 6489 patients with COVID-19 from various countries, we found that the administration of tocilizumab was not associated with a reduction in all-cause mortality compared to placebo or standard of care. The effect was also not significant in patients who required ICU admission at baseline. However, drug use was associated with a lesser need for ICU admission but a higher risk of secondary infections.

This analysis included all published RCTs to date regarding tocilizumab usage for COVID-19. Due to the non-availability of individual patient data, subgroup analyses based upon age, gender, or timing of tocilizumab initiation were not performed. All these parameters may affect individual patient outcomes. The inclusion criteria of different trials varied, and some trials included more critical patients while others excluded them. Also, the baseline severity scores such as SOFA (Sequential Organ Failure Assessment) or APACHE (Acute Physiology and Chronic Health Evaluation) were not available for all studies. Due to this, it was not possible to further categorize the trial patients based upon disease severity (other than the ICU admission at recruitment). A previously published meta-analysis, which did not include any of the RCTs also demonstrated no definite benefit; however, a hint towards safety concerns was found from the studies which included a control group [14].

The findings of this meta-analysis suggest that the use of tocilizumab is not associated with reduced mortality in COVID19; however, it should be noted that there is a higher risk of secondary infections, which is not the case with the use of steroids in severe COVID-19, where it has been seen that steroids reduce mortality without a higher risk of nosocomial infections but have concerns of hyperglycemia $[23,24]$. We did not analyze the complete adverse effects of the therapy due to the non-availability of complete data; however, the issue of secondary infections remains a major concern with tocilizumab use. Another important issue while considering use of tocilizumab is the type of inflammation in patients with COVID-19. It has been shown in a retrospective study that among various subtypes of cytokine storm syndrome, the macrophage activation syndrome subtype has better response 
to tocilizumab as compared to cytokine release syndrome [25]. Due to non-availability of individual patient data, we could not perform such analysis to identify individuals who may respond better to tocilizumab therapy. After the analysis of this meta-analysis, three more RCTs have been published regarding the use of tocilizumab in severe COVID-19. One trial randomly assigned 434 patients to tocilizumab plus remdesivir and 215 to placebo plus remdesivir. Among these, $78(18.2 \%)$ and $42(19.7 \%)$ patients died by day 28 in tocilizumab and placebo groups, respectively $(\mathrm{p}=0.69)$ [26]. The study by Hamed et al. randomized 23 patients to steroids alone and 26 patients to steroids with tocilizumab and found no difference in mortality at day $45(4.35 \%$ vs $7.65 \%$, respectively) [27]. The third trial (COV-AID) randomized the patients to IL-1 as well as IL-6 inhibition as compared to placebo [28]. In this study, the mortality was not different between patients who received tocilizumab as compared to usual care $[10 / 81(12 \%)$ and 9/74 (12\%), respectively]. This meta-analysis of randomized controlled trials has summarized all the published RCTs regarding the use of tocilizumab therapy in COVID-19. The trials included in the meta-analysis were conducted in different geographic areas and provide generalizable evidence. The protocol for the analysis was published publically on the PROSPERO database before initiation to avoid post-search bias.
This meta-analysis has several limitations, as well. We included only the published peer-reviewed randomized controlled trials, and ongoing partially completed trials, as well as non-peer-reviewed preprint articles, were not included. We did not report and analyzed the use of additional therapies used in most trials in varying numbers of patients. Among these therapies, steroids may be the reason behind no benefit due to tocilizumab. We assessed the side-effect profile in the form of secondary infections only rather than all sideeffects of tocilizumab therapy in these patients. As all the trials included adults only, we cannot make any conclusions regarding the utility of tocilizumab therapy in children with severe COVID-19. One trial reported 21-day mortality, which was used for calculations, potentially leading to inconsistency and under-reporting as delayed deaths due to severe COVID-19 are well reported.

\section{Conclusions}

This meta-analysis of randomized controlled clinical trials of administration of tocilizumab compared to placebo or standard of care demonstrated a statistically non-significant reduction in mortality, with higher odds of secondary infections.

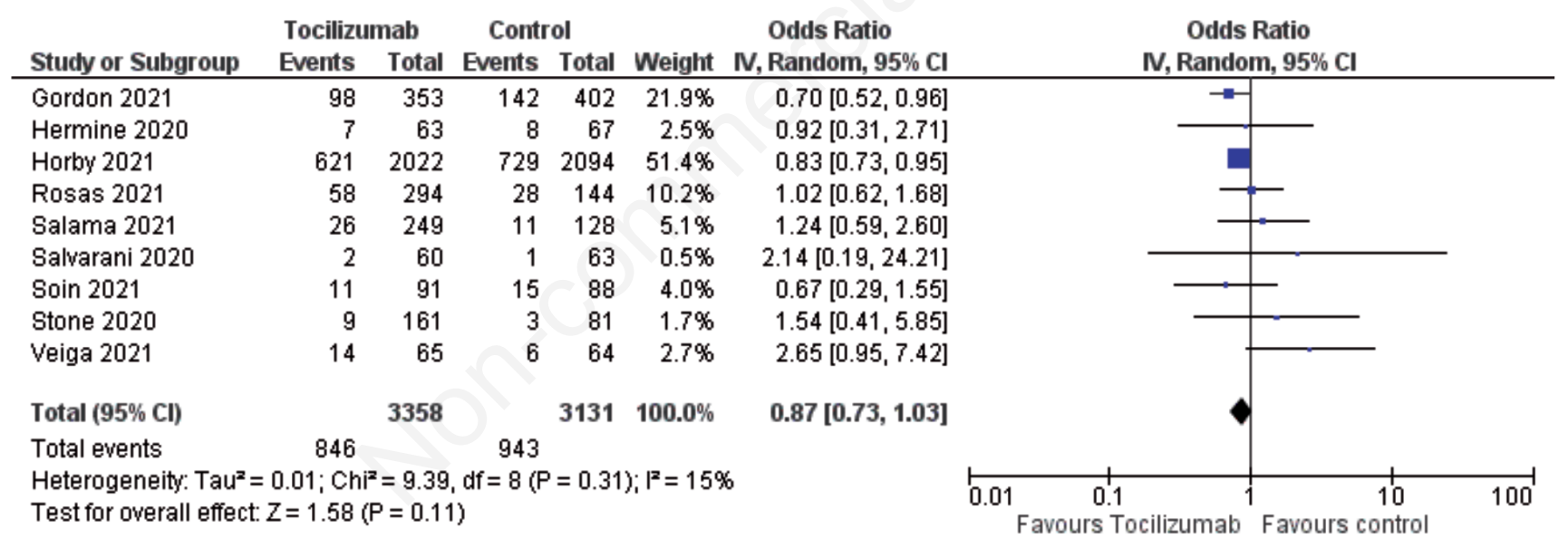

Figure 3. The pooled analysis of all-cause mortality in each trial.

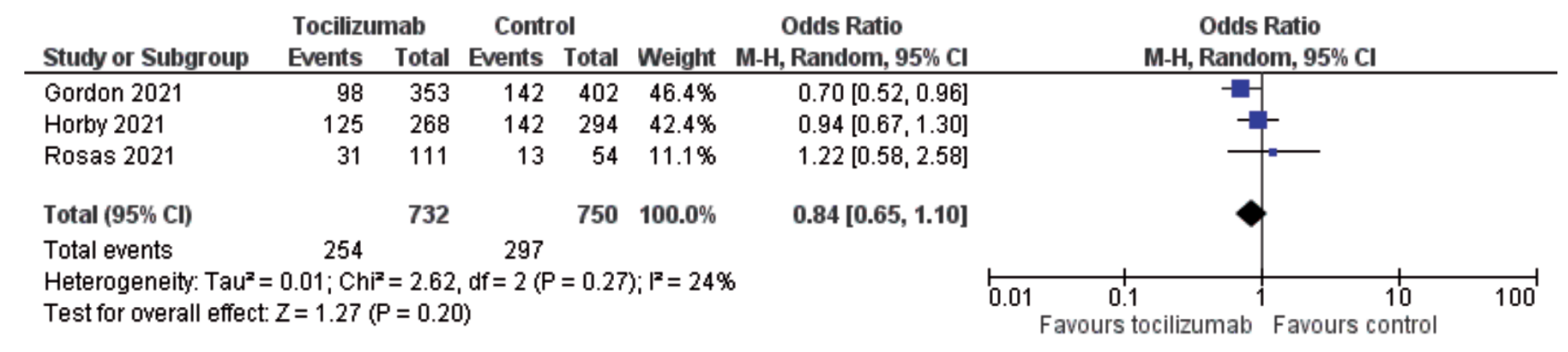

Figure 4. The pooled analysis for the all-cause mortality among patients admitted to ICU at enrollment. 


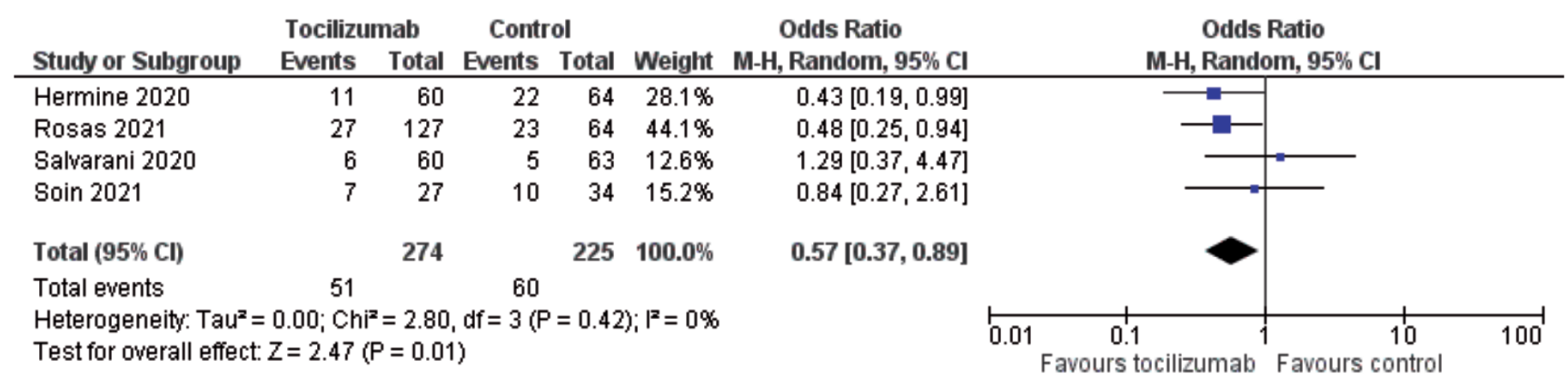

Figure 5. The pooled analysis for need for ICU admission among patients not admitted to ICU at enrollment.

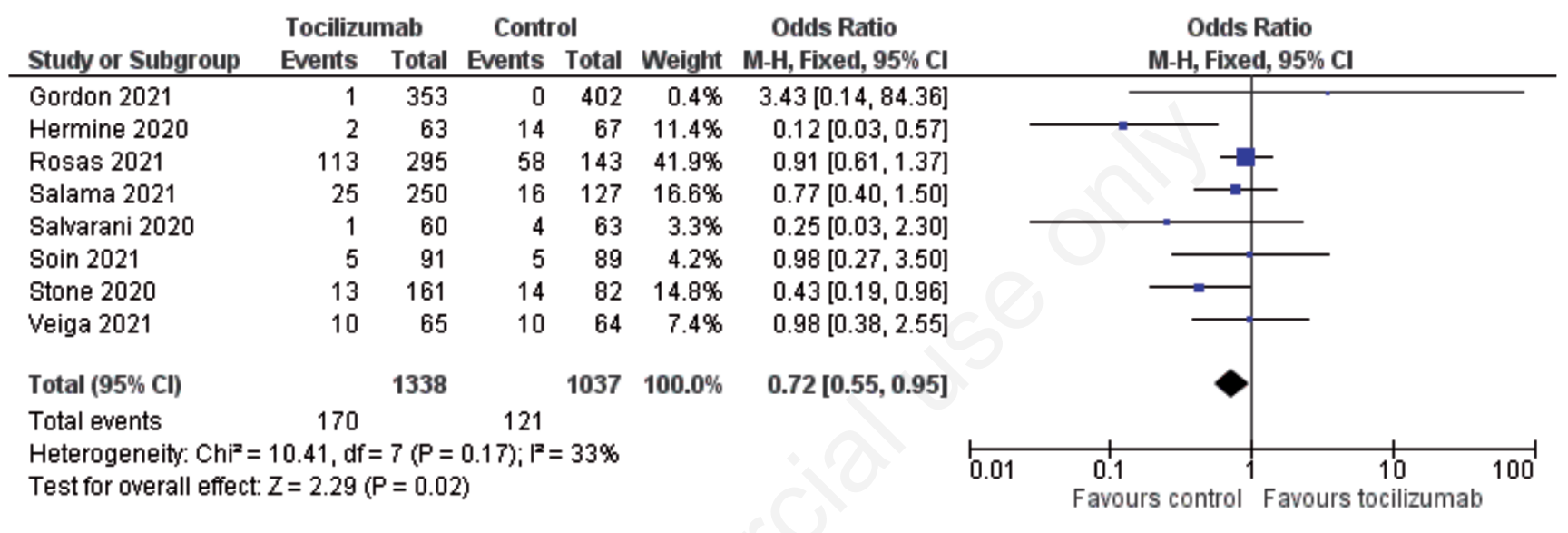

Figure 6. The pooled analysis of secondary infections in the both groups in the eight trials.

\section{References}

1. Aggarwal N, Boppana TK, Mittal S. COVID-19 pandemic: The testing times for healthcare workers. Monaldi Arch Chest Dis 2021;91:1515.

2. Sehgal IS, Guleria R, Singh S, et al. A randomised trial of Mycobacterium $w$ in critically ill patients with COVID-19: ARMY-1. ERJ Open Research 2021;7:00059-2021.

3. Boppana TK, Mohan A, Madan K, et al. Candidate immunomodulators for COVID-19: Heat-killed Mycobacterium w and BCG vaccine. Adv Respir Med 2021;89:350-1.

4. Sryma PB, Mittal S, Madan K, et al. Reinventing the wheel in ARDS: Awake proning in COVID-19. Arch Bronconeumol 2020;56:747-9.

5. Sryma P, Mittal S, Madan K, et al. Awake prone positioning in non-intubated patients for the management of hypoxemia in COVID-19: A systematic review and meta-analysis. Monaldi Arch Chest Dis 2021;91:1623.

6. Li P, Lu Z, Li Q, et al. Administration timing and efficacy of tocilizumab in patients With COVID-19 and elevated IL-6. Front Mol Biosci 2021;8:651662.

7. Gritti G, Raimondi F, Bottazzi B, et al. Siltuximab downregulates interleukin- 8 and pentraxin 3 to improve ventilatory status and survival in severe COVID-19. Leukemia 2021;2710-4

8. Corominas H, Castellví I, Diaz-Torné C, et al. Sarilumab (IL-6R antagonist) in critically ill patients with cytokine release syndrome by SARS-CoV2. Medicine (Baltimore) 2021;100:e25923.

9. RECOVERY Collaborative Group. Tocilizumab in patients admitted to hospital with COVID-19 (RECOVERY): a randomised, controlled, open-label, platform trial. Lancet 2021;397:1637-45.

10. Hermine O, Mariette X, Tharaux P-L, et al. Effect of tocilizumab vs usual care in adults hospitalized with COVID19 and moderate or severe pneumonia: A randomized clinical trial. JAMA Intern Med 2021;181:32-40.

11. Salama C, Han J, Yau L, et al. Tocilizumab in patients hospitalized with Covid-19 pneumonia. N Engl J Med 2021;384:20-30.

12. Salvarani C, Dolci G, Massari M, et al. Effect of tocilizumab vs standard care on clinical worsening in patients hospitalized with COVID-19 pneumonia: A randomized clinical trial. JAMA Intern Med 2021;181:24-31.

13. Elavarasi A, Sahoo RK, Seth T, et al. Anti-interleukin-6 therapies for Covid-19: A systematic review, critical appraisal and meta-analysis. Natl Med J India 2020;33:152-7.

14. Cortegiani A, Ippolito M, Einav S. Rationale and evidence on the use of tocilizumab in COVID-19: A systematic review. Pulmonology 2021;27:52-66.

15. Liberati A, Altman DG, Tetzlaff J, et al. The PRISMA statement for reporting systematic reviews and meta-analyses of studies that evaluate healthcare interventions: explanation and elaboration. BMJ 2009;339:b2700-b2700. 
16. Mariette X, Hermine O, Tharaux P-L, et al. Effectiveness of tocilizumab in patients hospitalized with COVID-19: A followup of the CORIMUNO-TOCI-1 randomized clinical trial. JAMA Intern Med 2021;181:1241-3.

17. Zhao H, Zhu Q, Zhang C, et al. Tocilizumab combined with favipiravir in the treatment of COVID-19: A multicenter trial in a small sample size. Biomed Pharmacother 2021;133:110825.

18. Gordon AC, Mouncey PR, Al-Beidh F et al. Interleukin-6 receptor antagonists in critically ill patients with Covid-19. N Engl J Med 2021;384:1491-502.

19. Soin AS, Kumar K, Choudhary NS, et al. Tocilizumab plus standard care versus standard care in patients in India with moderate to severe COVID-19-associated cytokine release syndrome (COVINTOC): an open-label, multicentre, randomised, controlled, phase 3 trial. Lancet Respir Med 2021;9:511-21.

20. Rosas IO, Bräu N, Waters M, et al. Tocilizumab in hospitalized patients with severe Covid-19 pneumonia. N Engl J Med 2021;384:1503-16.

21. Stone JH, Frigault MJ, Serling-Boyd NJ, et al. Efficacy of tocilizumab in patients hospitalized with Covid-19. N Engl J Med 2020;383:2333-44.

22. Veiga VC, Prats JAGG, Farias DLC, et al. Effect of tocilizumab on clinical outcomes at 15 days in patients with severe or critical coronavirus disease 2019: randomised controlled trial. BMJ 2021;372:n84.

23. WHO Rapid Evidence Appraisal for COVID-19 Therapies (REACT) Working Group, Sterne JAC, Murthy S, et al. Association between administration of systemic corticosteroids and mortality among critically ill patients with COVID19: A meta-analysis. JAMA 2020;324:1330-41.

24. Mittal S, Madan K, Mohan A, et al. Diabetes in COVID-19: Steroid effect. J Med Virol 2021;93:4166.

25. Oliynyk O, Barg W, Slifirczyk A, et al. Efficacy of tocilizumab therapy in different subtypes of COVID-19 cytokine storm syndrome. Viruses 2021;13:1067.

26. Rosas IO, Diaz G, Gottlieb RL, et al. Tocilizumab and remdesivir in hospitalized patients with severe COVID-19 pneumonia: a randomized clinical trial. Intensive Care Med 2021;47:1258-70.

27. Hamed DM, Belhoul KM, Al Maazmi NA, et al. Intravenous methylprednisolone with or without tocilizumab in patients with severe COVID-19 pneumonia requiring oxygen support: A prospective comparison. J Infect Public Health 2021;14:985-9.

28. Declercq J, Van Damme KFA, De Leeuw E, et al. Effect of anti-interleukin drugs in patients with COVID-19 and signs of cytokine release syndrome (COV-AID): a factorial, randomised, controlled trial. Lancet Respir Med 2021;9:1427-38. 